\title{
Probing the Intrinsic Bending Stiffness of 2D Multilayers and Heterostructures Using Aberration-corrected STEM
}

Edmund Han ${ }^{1}$, Jaehyung $\mathrm{Yu}^{2}$, Mohammad Houssain ${ }^{2}$, Kenji Watanabe ${ }^{3}$, Takashi Taniguchi ${ }^{3}$, Elif Ertekin $^{2}$, Arend van der Zande ${ }^{2}$ and Pinshane Huang ${ }^{2}$

${ }^{1}$ University of Illinois at Urbana-Champaign, Champaign, Illinois, United States, ${ }^{2}$ University of Illinois at Urbana-Champaign, Urbana, Illinois, United States, ${ }^{3}$ National Institute for Materials Science, Tsukuba, Ibaraki, Japan

Because they can be isolated down to single unit cells and recombined almost arbitrarily through layerby-layer stacking, two-dimensional (2D) materials offer a unique opportunity to study the structureproperty relationship of materials with high precision using electron microscopy. Due to their atomically thin structure, 2D materials are predicted to be among the most flexible, high-quality electronic materials known. However, efforts to experimentally measure their bending stiffness, using conventional techniques such as nanoindentation and electrostatic actuation, have yielded conflicting results that range over orders of magnitude [1-3]. Here, we show that electron microscopy is an exceptionally precise, yet flexible technique for measuring the intrinsic bending stiffness of $2 \mathrm{D}$ materials. We use these techniques to quantify the bending stiffness and probe the atomic scale bending mechanisms for graphene, $\mathrm{MoS}_{2}$, and their heterostructures.

We design and fabricate 2D nanostructures specifically for mechanical properties measurements in the scanning transmission electron microscope (STEM). These structures, which consist of 2D materials or multilayers draped over atomic steps of hexagonal boron nitride ( $\mathrm{h}-\mathrm{BN})$, encode the bending stiffness in the conformation of the layers in cross-sectional STEM images (Figure 1a-d). We obtain bending stiffness values of $1.4 \mathrm{eV}$ for monolayer graphene [5] and $10.5 \mathrm{eV}$ for monolayer $\mathrm{MoS}_{2}$, consistent with density functional theory (DFT) predictions. For multilayers of Bernal-stacked graphene and $2 \mathrm{H} \mathrm{MoS}_{2}$, we find that the bending stiffness varies strongly as a function of bending angle, tuning by almost $400 \%$ for trilayer graphene (Figure 1e). This unusual behavior results from the atomic scale bending mechanism in 2D multilayers, which is dominated by interlayer shear and slip [5].

Our findings have profound implications on 2D heterostructures, where we demonstrate that the bending stiffness can be controlled by tailoring the interfacial interactions between vertical stacks of 2D materials. We investigated heterostructures comprised of two $\mathrm{MoS}_{2}$ layers and two graphene layers in different stacking arrangements (Figure 2a-b). We show that, by simply changing the stacking order of the heterostructure, the bending stiffness can be tuned from $22 \mathrm{eV}$ to $80 \mathrm{eV}$ (Figure 2c). We achieve the lowest bending stiffness values by alternating layers of graphene and $\mathrm{MoS}_{2}$, where each layer is separated by incommensurate, low-friction heterointerfaces. Using a combination of DFT and continuum mechanics, we produce a model to predict the bending stiffness of arbitrary 2D heterostructures. Together, our results provide a new lower limit for the fabrication of ultra-soft, high mobility electronics and demonstrate new methods to fabricate 2D heterostructures with tailored bending stiffnesses [6]. 

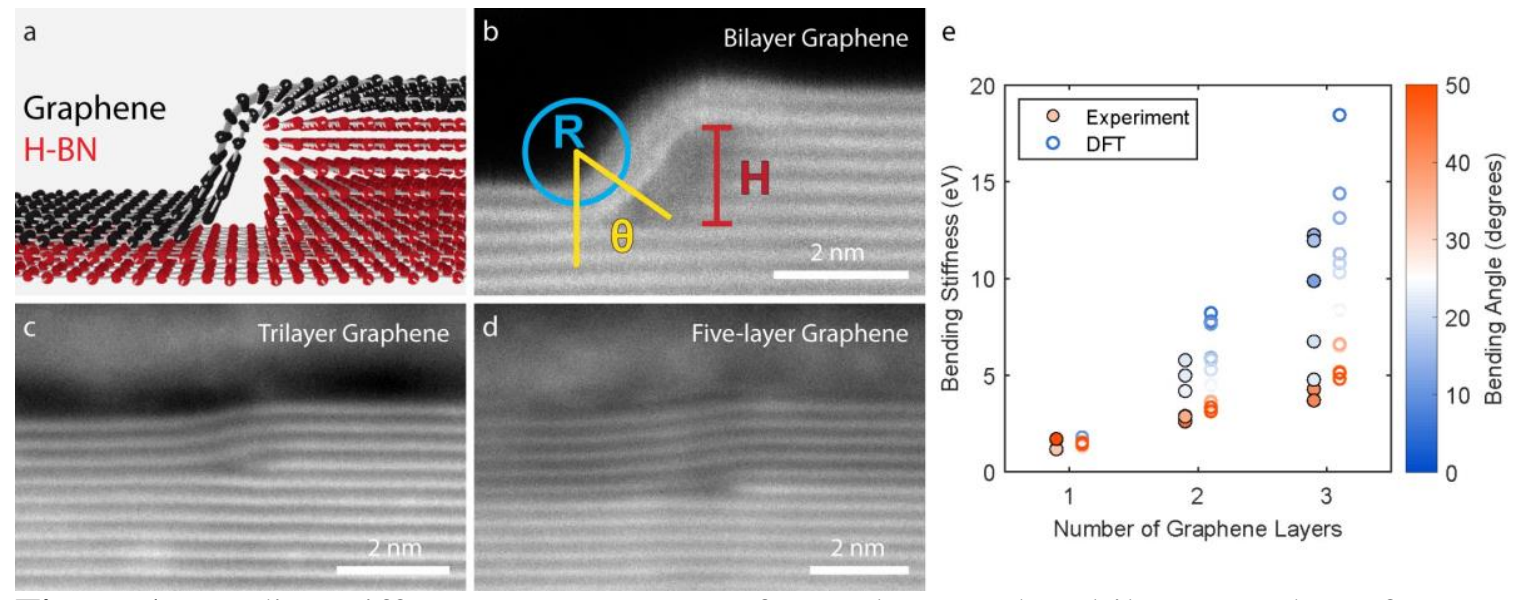

Figure 1. Bending stiffness measurements of monolayer and multilayer graphene from annular dark field (ADF-) STEM images. a) Schematic of bilayer graphene on a step of hexagonal boron nitride (h-BN). bd) ADF-STEM images of multilayer graphene draped over h-BN steps. We derived a mechanical model that relates the bending stiffness to geometric parameters, including the radius of curvature, bending angle, and step height, measured from STEM images. e) Experimental and DFT calculations of bending stiffness for 1-4 layer graphene. Data points are color-coded via bending angle. Both sets show strong quantitative agreement and a clear bending angle dependence of bending stiffness for multilayer graphene. Adapted from Reference [5].

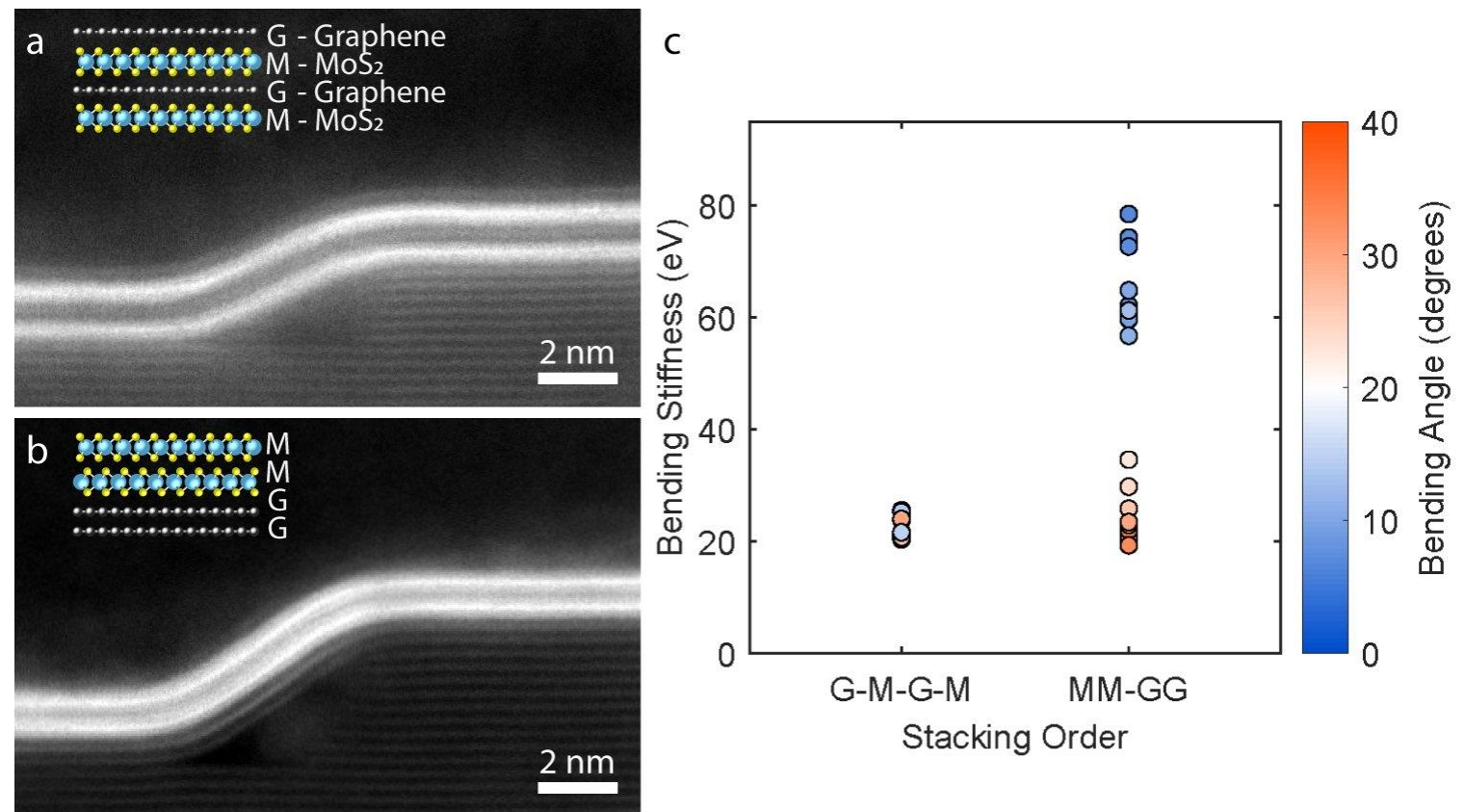

Figure 2. Bending stiffness measurements of 2D heterostructures from ADF-STEM images. a-b) ADFSTEM images of graphene-MoS2 heterostructures draped over atomic steps of h-BN. Each heterostructure consists of the same four layers--two graphene $(\mathrm{G})$ and two MoS2 (M) layers--in different stacking orders. c) Experimental calculations of bending stiffness for each structure. Data points are color-coded via bending angle. GMGM, where every interface is an incommensurate heterointerface, has a low bending $\sim 20 \mathrm{eV}$ at all bending angles. 


\section{References}

[1] Akinwande, D., et al. Extreme Mechanics Letters 13, $42-77$ (2017).

[2] Poot, M., and van der Zant, H. S. J. Applied Physics Letters 92, 063111 (2008).

[3] Lindahl, N., et al. Nano Letters 12, 3526-3531 (2012).

[4] Wei, Y., et al. Nano Letters 13, 26-30 (2013).

[5] Han, E. and Yu, J., et al. Nature Materials (2019). doi: 10.1038/s41563-019-0529-7

[6] This work was supported by NSF-MRSEC award no. DMR-1720633 and the Materials Research Laboratory Central Facilities at the University of Illinois. 